\title{
Avaliação da Disponibilizaçâo de Kits de Redução de Danos '
}

\section{An Evaluation of Harm Reduction Kits Distrubution '}

\author{
Fabiana Delbon \\ Psicóloga Especialista em Dependência Química - UNIFESP e em \\ Terapia de Casal e de Família - PUC/SP. Consultora Técnica da \\ UNESCO junto a Secretaria Municipal de Saúde da cidade de São \\ Paulo. \\ E-mail: fdelbonळprefeitura.sp.gov.br \\ delbon_2000®yahoo.com.br

\section{Vera Da Ros} \\ Mestre e Psicóloga - Consultora em Drogas e Aids - Ministério da \\ Saúde/PN-DST/AIDS. Especialista em Gerência e Avaliação de \\ Projetos pelo PROAD/UNIFESP. Membro da Diretoria Executiva da \\ ONG DINAMO. \\ E-mail: veradarosळhotmail.com, info®dinamo.org.br

\section{Elza Maria Alves Ferreira} \\ Psicóloga Especialista em Saúde Pública - UNAERP, Coordena- \\ dora do Projeto Redução de Danos da cidade de São Paulo - PRD \\ Sampa e Consultora Técnica da UNESCO \\ Pesquisadora NEPAIDS - Projeto desenvolvido em Redução de \\ Danos. \\ E-mail: eferreiraळprefeitura.sp.gov.br \\ I Este artigo é baseado em relatório original publicado em in- \\ glês pela Organização Mundial da Saúde - Centro de Kobe, Ja- \\ pão WHO/WKC/Tech. Ser./05/14, intitulado An Evaluation of the \\ Harm Reduction Project in São Paulo. A Universidade de São Paulo \\ é responsável por esta versão resumida em português. \\ I This material is based on an original English report published \\ by WKC, intitled "An evaluation of the harm reduction project \\ in São Paulo". The University of São Paulo is solely responsible \\ for this Portuguese summary.
}

\section{Resumo}

A parceria com a Faculdade de Saúde Pública da Universidade de São Paulo e Organização Mundial da Saúde - KOBE Center possibilitou que a Prefeitura do Município de São Paulo investigasse as intervenções realizadas junto à população de usuários de drogas e junto aos serviços públicos municipais dirigidos a essa população, durante o período de julho de 2003 a novembro de 2004. Pesquisou-se dessa forma a implantação de estratégias de redução de danos em serviços de tratamento a usuários de drogas. A estratégia de avaliação das intervenções focou a distribuição de kits de redução de danos em serviços de tratamento da dependência química, os Centros de Atenção Psicossocial Álcool e Drogas da área temática de Saúde Mental do município de São Paulo - CAPS ad - cujos técnicos foram treinados pelos profissionais do projeto PRD Sampa, visando a implantação da Política de Redução de Danos. A introdução do conceito de redução de danos (RD) nos CAPS ad fez com que se compreendesse a complexidade da tarefa e o impacto no serviço e no território de abrangência. Após tal constatação, desenharam-se treinamentos e reuniões de fortalecimento para sensibilização dos profissionais, usuários, familiares e comunidade.

Palavras-chave: Redução de danos; Tratamento da dependência química; Políticas públicas de saúde. 


\section{Abstract}

The PRD Sampa (the Sampa Harm Reduction Project) was initiated in the Mental Health District of São Paulo to implement a harm reduction policy for drug and alcohol users in the city and reduce their exposure to sexually transmitted diseases - STDs and HIV. Sao Paulo Alcohol and Drug Psycho-social Care Centers (CAPS ad) run by the Mental Health Division of Sao Paulo municipality dispatched their staff to receive professional training from PRD Sampa to implement Harm Reduction Public Policy. The study was implemented from July 2003 to November 2004 in São Paulo, in order to evaluate the interventions. The presentation of the harm reduction (HR) concept at CAPS ad shed light on the complexity of the task and the impact of the service within the territory covered. After observation, training and booster meetings were designed to raise the awareness of professionals, users, families and the community.

Keywords: Harm Reduction; Drug Abuse Treatment; Public Health Policy.

\section{Antecedentes: Ética, Direitos Humanos e Cidadania}

A Declaração Universal dos Direitos Humanos, adotada cinqüenta anos atrás para todos os povos e todas as nações, determina que "todos os indivíduos, como membros da sociedade, têm direito à segurança social e à concretização (...) dos direitos econômicos, sociais e culturais indispensáveis a sua dignidade e ao livre desenvolvimento de sua personalidade" (Art. 22).

O respeito aos direitos humanos e a realização dos objetivos de saúde pública são complementares. A Constituição da Organização Mundial da Saúde, em 1946, define como saúde: um estado de completo bemestar físico, mental e social e não, simplesmente, a ausência de doença ou enfermidade. Tal Constituição declara que a busca do melhor padrão possível de saúde é um dos principais direitos de todo ser humano, sem distinção de raça, posição política, religião, condição econômica ou social.

Importantes metas globais do Sistema das Nações Unidas, até 2020 , buscam que: “... o ônus mundial das doenças será significativamente reduzido. Isso será alcançado pela implementação de bons programas de controle de doenças que busquem a reversão da atual tendência de aumento da incidência de tuberculose, HIV/AIDS (...) - e da incapacidade causada por elas que todos os países terão introduzido e estarão administrando e controlando ativamente, estratégias que fortaleçam estilos de vida promotores de saúde e enfraqueçam os prejudiciais, mediante uma combinação de programas comunitários reguladores, econômicos, educacionais e organizacionais" (grifo dos autores) (ONU, 2000).

A realidade brasileira tem demonstrado que, apesar do caráter universal dos direitos afirmados na Declaração, sua efetivação não depende exclusivamente de seu reconhecimento. Ao contrário, depende de uma luta cotidiana cuja responsabilidade é de todos e de cada um de nós.

Segundo o Manual de Redução de Danos (BRASIL, 2001 a), a consciência ética é a nossa capacidade de reconhecer no outro - ainda que ele seja diferente - a nossa própria humanidade. A luta cotidiana pela efetivação dos direitos humanos e de cidadania, nada mais é do que a luta pela efetivação dos nossos pró- 
prios direitos enquanto membros da comunidade humana. Quando aceitamos que os direitos de uma pessoa sejam violados, na verdade, do ponto de vista ético, estamos aceitando uma situação que ameaça o direito de todos.

No caso específico da redução de danos, quando uma sociedade não reconhece os direitos de uma pessoa que faz uso de uma droga ilícita, significa que essa sociedade, do ponto de vista ético, está afirmando que umas pessoas são mais cidadãs do que outras. Portanto, a condição de cidadão passa a ser secundária em relação à de usuário de droga, ou seja, primeiro a pessoa é julgada por fazer uso de droga e, em decorrência disso, perde o reconhecimento de sua condição de cidadão.

\section{Redução de danos no Mundo}

A posição oficial das agências das Nações Unidas em relação às políticas e estratégias de prevenção da transmissão do HIV/AIDS entre usuários de drogas está definida no documento "Posição Oficial do Sistema das Nações Unidas, Prevenção da Transmissão do HIV entre Usuários de Drogas" (ONU, 20oo). O documento reconhece a eficácia dos programas de troca de seringas e agulhas ao apontar "a redução dos comportamentos de risco quanto ao uso de agulhas e à transmissão do HIV" sem a criação de "evidências sobre o aumento do uso de drogas”.(item 10, p.2)

O Sistema ONU também afirma, por meio do documento, que os benefícios dos programas de redução de danos "aumentam consideravelmente quando vão além da troca de seringas e incluem educação quanto à Aids, o aconselhamento e o encaminhamento para uma variedade de opções de tratamento" (item 10, p.2), referindo-se às várias opções de tratamento da dependência química.

A luta contra a Aids tem mobilizado progressivamente os governos, organizações internacionais e organizações não governamentais mas, a despeito dos esforços maciços para informar o público sobre os riscos, as mudanças de comportamento não seguiram como previsto. A infecção continua em expansão rápida e há questões sobre a eficácia dos esforços de combate à epidemia. A experiência demonstrou que o HIV e a Aids são fenômenos complexos, com aspectos múltiplos e que demandam uma cooperação interinstitucional e estratégias multidimensionais.

\section{Ações programáticas estratégicas do Ministério da Saúde}

A exclusão social e a ausência de cuidados que atingem, de forma histórica e contínua, aqueles que sofrem de transtornos mentais, apontaram para a necessidade da reversão de modelos assistenciais, de modo a contemplar as reais necessidades da população, o que implica a disposição para atender igualmente ao direito de cada cidadão. Tal lógica também deve ser contemplada no planejamento de ações voltadas para a atenção integral às pessoas que consomem álcool e outras drogas, por ser grave problema de saúde pública no país e encontrar ressonância nos diversos segmentos da sociedade, pela relação comprovada entre o consumo e os agravos sociais que dele decorrem ou que o reforçam, já que, no Brasil:

$3 \%$ da população geral sofre com transtornos mentais severos e persistentes;

$6 \%$ da população apresenta transtornos psiquiátricos graves decorrentes do uso de álcool e outras drogas;

$12 \%$ da população necessita de algum atendimento em saúde mental, seja ele contínuo ou eventual;

2,3\% do orçamento anual do SUS é destinado à Saúde Mental (Saúde Mental - Brasil, Ministério da Saúde)

O enfrentamento desta problemática constitui uma demanda mundial. De acordo com a Organização Mundial de Saúde, cerca de 10\% das populações dos centros urbanos de todo o mundo consomem abusivamente substâncias psicoativas, independentemente da idade, sexo, nível de instrução e poder aquisitivo. Salvo variações sem repercussão epidemiológica significativa, esta realidade encontra equivalência em território brasileiro.

No vácuo de propostas concretas e na ausência do estabelecimento de uma clara política pública de saúde voltada para este segmento, surgiram no Brasil alternativas de tratamento de caráter rígido, fechado e tendo a abstinência como único objetivo a ser alcançado.

Historicamente, a questão do uso abusivo e/ou dependência de álcool e outras drogas tem sido abordada por uma ótica predominantemente psiquiátrica ou médica. As implicações sociais, psicológicas, econômicas e políticas são evidentes, e devem ser consideradas na compreensão global do problema. Cabe ainda destacar que o tema vem sendo associado à 
criminalidade e à oferta de tratamentos organizados em modelos de exclusão, no sentido de afastar os usuários de drogas do convívio social.

No campo da política de atenção integral em álcool e outras drogas no Brasil, vimos que o tema tem sido tratado de modo pontual, contando com esforços de setores e grupos preocupados com o aumento exponencial do problema. É importante destacar que o Ministério da Saúde tem dentre suas atribuições o desafio de prevenir, tratar, reabilitar os usuários de álcool e outras drogas como um problema de saúde pública. Esta decisão atende às propostas que foram enfaticamente recomendadas pela III Conferência Nacional de Saúde Mental, em dezembro de 2001. (BRASIL, 2001a).

Comprometer-se com a formulação, execução e avaliação de uma política de atenção a usuários de álcool e outras drogas exige exatamente a ruptura de uma lógica binarizante que separa e detém o problema em fronteiras rigidamente delineadas, e cujo eixo principal de entendimento (e, portanto, de tratamento) baseia-se na associação drogas-comportamento anti-social (álcool) ou criminoso (drogas ilícitas). Em ambos os casos, há um único objetivo a ser alcançado: a abstinência. A ruptura dessa lógica é a meta da Política de Atenção Integral em Álcool e outras Drogas do Ministério da Saúde.

Desta forma, as propostas de um Programa de Álcool e Drogas têm o objetivo imprescindível de:

- Alocar a questão do uso de álcool e outras drogas como problema de saúde pública;

- Indicar o paradigma da redução de danos - estratégia de saúde pública que visa reduzir os danos causados pelo abuso de drogas lícitas e ilícitas, resgatando o usuário em seu papel autoregulador, sem a preconização imediata da abstinência e incentivando-o à mobilização social - nas ações de prevenção e de tratamento, como um método clínico-político de ação territorial inserido na perspectiva da clínica ampliada;

- Formular políticas que possam desconstruir o senso comum de que todo usuário de droga é um doente que requer internação, prisão ou absolvição;

- Mobilizar a sociedade civil, oferecendo condições de exercer seu controle, participar das práticas preventivas, terapêuticas e reabilitadoras, bem como estabelecer parcerias locais para o fortalecimento das políti- cas municipais e estaduais (Brasil, 2004-a, p.25).

As ações de redução de danos priorizaram a prevenção e o diagnóstico do HIV, por meio de intervenções de troca e distribuição de agulhas e seringas e deram visibilidade aos usuários de drogas injetáveis (UDIs) no SUS - Sistema Único de Saúde. Tais ações promoveram a organização de profissionais e usuários e trouxeram contribuições significativas para a revisão das leis em vigor. Proporcionaram, também, o compartilhamento de saberes técnicos e saberes populares, ao criar condições para a construção de estratégias eficazes na abordagem dos problemas de saúde dos consumidores de drogas.

A estratégia de redução de danos desenvolvida entre UDIs pode ser estendida a usuários de outras drogas, tais como álcool, crack, tabaco, tanto no tocante à disponibilização de insumos para uso seguro, quanto no fortalecimento do protagonismo destes consumidores para a realização de ações entre pares e sua participação na formulação de políticas públicas, estratégias de comunicação e elaboração de materiais educativos.

\section{Tratamento da dependência de drogas e redução de danos}

A abstinência não pode ser o único objetivo a ser alcançado. Aliás, quando se trata de cuidar de vidas humanas, temos que, necessariamente, lidar com as singularidades, com as diferentes possibilidades e escolhas que são feitas. As práticas de saúde, em qualquer nível de ocorrência, devem levar em conta esta diversidade. Devem acolher, sem julgamento, o que em cada situação e com cada usuário - é possível, é necessário, está sendo demandado, pode ser ofertado, deve ser feito na busca de sua participação e o seu engajamento.

A abordagem da redução de danos nos oferece um caminho promissor por reconhecer cada usuário em sua singularidade, traçar com ele estratégias para promover a saúde e garantir seus direitos enquanto cidadão. E tratar significa possibilitar maior liberdade e co-responsabilidade daquele que está se tratando.

Tal abordagem favorece também que o usuário de droga injetável (UDI) quando vinculado a alguma ação de saúde tenha melhores indicadores comportamentais do que um UDI não acessado por ações de redução de danos: 
Tabela I - Principais indicadores comportamentais entre Usuários de Drogas Injetáveis - UDIs acessados e não acessados por ações de redução de danos: $N=869$ UDI em 7 cidades

\begin{tabular}{l|c|c}
\hline Indicadores & Clientes N = 599 & Não Clientes N $=270$ \\
\hline Soroprevalência positiva para o HIV & 39,3 & 29,9 \\
\hline Seringas e agulhas obtidas no PRD & 86,3 & 26,1 \\
\hline Intercâmbio de seringas /agulhas nos últimos 6 meses & 40,6 & 50,5 \\
\hline Descarte inadequado no último mês & 75,2 & 100 \\
\hline Tratamento de saúde nos últimos 6 meses & 82,6 & 74,2 \\
\hline Exame do HIV na vida & 66,4 & 55,8 \\
\hline Uso do preservativo nos últimos 6 meses & 42,3 & 31,1
\end{tabular}

Fonte: Caiaffa -Projeto Ajude Brasil - 2001

Quando se propõem discussões conceituais sobre RD é necessário que se considere a questão: danos para quem e para que? A resposta quase sempre abrange os danos que o uso de drogas provoca para indivíduos, famílias e vizinhos entre as classes trabalhadoras e entre os mais pobres, ou seja, aqueles que se utilizam do serviço público de saúde.

Muitos serviços para usuários de drogas sejam de tratamento, cuidados médicos em geral ou de suporte social, de uma maneira ou de outra se baseiam na proposição de que o usuário tem de parar o seu consumo. Trata-se de um dogma bem estabelecido. As leis sobre drogas são intolerantes - a abstinência ou o rigor da lei - e como contraponto a RD pode ser descrita como tolerante - ou melhor, como respeitosa, por ser baseada nos princípios de respeito aos usuários de drogas e, tal respeito, dá lugar a um diálogo honesto.

\section{Modelos de atenção - CAPS e redes assistenciais}

O Centro de Atenção Psicossocial (CAPS) é um serviço comunitário que tem como papel cuidar de pessoas que sofrem com transtornos mentais, em especial os transtornos severos e persistentes, no seu território de abrangência. A atenção deve incluir ações dirigidas aos familiares e à reinserção social dos usuários.

0 tratamento oferecido pelos CAPS deve ser singular, respeitando-se diferenças regionais, contribuições técnicas dos integrantes de sua equipe, iniciativas locais de familiares e usuários e articulações intersetoriais que potencializem suas ações.

O CAPS ad (Centro de Atenção Psicossocial - Álcool e Drogas) tem como especificidade de atenção o usuário de álcool e outras drogas. Uma das possibilidades de tratamento é trabalhar, junto a usuários e familiares, os fatores de proteção para o uso e dependência de substâncias psicoativas. Ao mesmo tempo deve-se buscar a minimização da influência dos fatores de risco para tal consumo e a diminuição do estigma e preconceito relativos ao uso de substâncias psicoativas, mediante atividades de cunho preventivo / educativo, através da lógica de redução de danos.

$\mathrm{O}$ uso destas medidas permite que sejam elaborados projetos terapêuticos mais flexíveis e de menor exigência, conseqüentemente adequados às necessidades de cada usuário dos serviços. Os CAPS ad atuam de forma articulada a outros dispositivos assistenciais em saúde mental (ambulatórios, leitos em hospital-geral, hospitais-dia) e da rede básica de saúde, bem como ao Programa de Saúde da Família e ao Programa de Agentes Comunitários de Saúde.

\section{Município de São Paulo}

Levantamentos epidemiológicos realizados em São Paulo apontam para uma prevalência anual dos transtornos mentais em torno de $\mathbf{2 0} \%$ da população. A maior parte dos pacientes sofre dos chamados transtornos mentais comuns (quadros depressivos e ansiosos, somatização) e de abuso ou dependência de álcool.

A Secretaria Municipal de Saúde conta com unidades especializadas no tratamento para usuários de álcool e outras drogas. A função principal desses serviços é prover assistência a especificidades dessa demanda através do atendimento ambulatorial individual, grupal, atendimento e orientação aos familiares, sempre respeitando o usuário e buscando melhorar sua qualidade de vida. Para isso foi fundamental a 
parceria entre as duas áreas temáticas Saúde Mental Álcool e Drogas e DST/AIDS pelo Programa de Redução de Danos da cidade de S.Paulo - PRD Sampa.

Nesses serviços de tratamento busca-se oferecer um acolhimento diferenciado, recursos para uso mais seguro, disponibilização de kits de redução de danos contendo: seringas, agulhas, swabs, preservativos, água destilada, copo plástico e material informativo. Constitui-se uma política de atendimento que trabalha com a realidade do fenômeno droga e de seus consumidores, suas necessidades e principalmente suas possibilidades, visando incluir e amparar socialmente o usuário de drogas.

Essa proposta faz emergir a figura do redutor de danos, que é o agente de saúde responsável pelo desenvolvimento dessa política, por ter a facilidade de penetração nos locais onde os usuários vivem ou fazem uso de drogas, que caracteriza o trabalho de pares. Freqüentemente são ex-usuários ou usuários na ativa e sendo assim podem obter um bom resultado no trabalho de prevenção por conhecerem a realidade dos UDIs e por serem respeitados em seu meio.

Diante do exposto e da necessidade de se ampliar as ações de prevenção à população de usuários de drogas e usuários de drogas injetáveis, a Secretaria Municipal de Saúde, por meio da integração de áreas já descrita, elaborou o projeto de "Treinamento e Capacitação de Profissionais dos Centros de Atenção Psicossocial Álcool e Drogas" com o objetivo de sensibilizar os profissionais para o acolhimento e disponibilização dos insumos de Redução de Danos.

\section{A Pesquisa e seus Objetivos}

Através de literatura especializada e pela experiência, sabia-se que a simples disponibilização de seringas não atingiria seu papel preventivo e, para tanto, vários recursos comunitários foram utilizados. Em decorrência dessa multiplicidade de ações, para determinação da eficácia do projeto foi fundamental a parceria com a Faculdade de Saúde Pública da Universidade de São Paulo e Organização Mundial da Saúde KOBE Centre, o que possibilitou que a Prefeitura do Município de São Paulo investigasse as intervenções realizadas junto à população de usuários de drogas e junto aos serviços públicos municipais dirigidos a essa população, durante o período de julho de 2003 a novembro de 2004.
Optou-se pela utilização de aplicação de metodologia quantitativa, no levantamento de dados relativos à distribuição de seringas e de metodologia qualitativa no trabalho de campo, direcionado à rede de usuários e profissionais da saúde.

Os objetivos do estudo:

- Sensibilizar e preparar os funcionários da rede de atendimento quanto ao acolhimento e disponibilização dos insumos de redução de danos.

- Avaliar a percepção tanto dos usuários das unidades de tratamento de álcool e drogas quanto dos profissionais que atuam nessas unidades em relação à distribuição dos kits e os reflexos desta nova prática na organização desses serviços.

- Expandir cursos de treinamento para agentes de saúde que lidam com usuários de drogas injetáveis.

A partir de 2001, todas as intervenções foram registradas e planilhas foram construídas permitindo análise e comparação dos dados, por tipo de serviço e por região da cidade.

\section{Gráfico I - Seringas distribuídas}

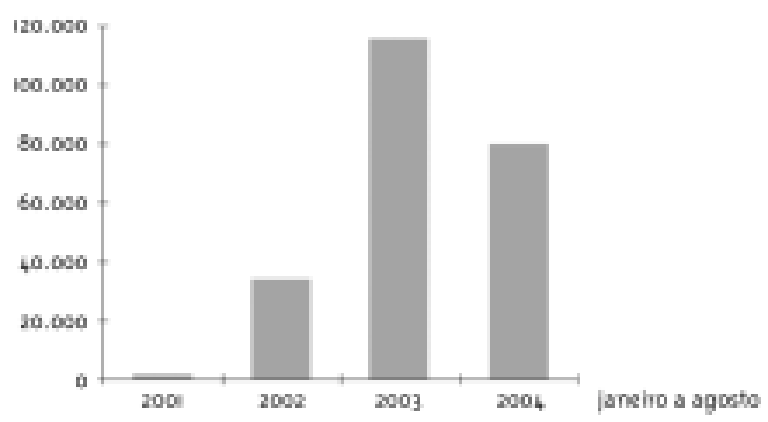

Fonte: PRD Sampa - Prefeitura Municipal de São Paulo

\section{Gráfico 2 - UDI acessados}

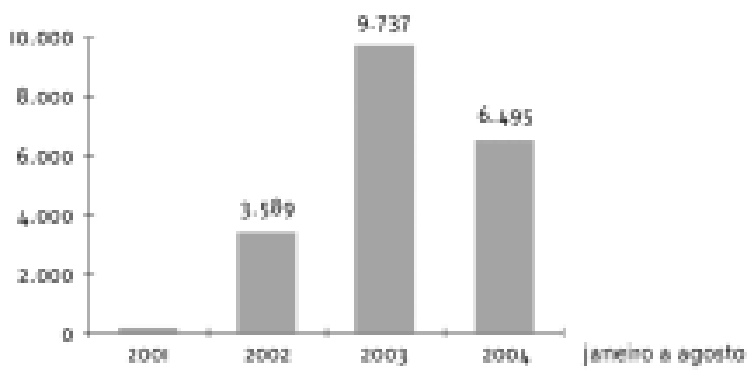

Fonte: PRD Sampa - Prefeitura Municipal de São Paulo 
É possível observar nos gráficos acima que o esforço de distribuição de seringas e kits está sendo eficaz no município de São Paulo. A adesão de vários serviços de saúde em Aids, Saúde Mental e de outras modalidades, como a atenção básica garante a continuidade desses esforços do PRD Sampa .

Com o PRD Sampa implantado e com treinamento das equipes, a população de UDIs torna-se visível: são quase 10.000 UDIs acessados em 2003 e os números de 2004 apontam para essa meta também.

Com a experiência nos serviços de Aids e com bons resultados atingidos, o processo deveria ser replicado nos serviços de tratamento da dependência quími- ca, numa tentativa de desconstrução de velhos conceitos, como: São Paulo não tem UDIs.

Os dados colhidos através de observação participante e grupos focais, portanto, de natureza qualitativa permitiram classificação das respostas por tópicos. Com o intuito de tornar os dados mais consistentes, sentiu-se a necessidade de consolidá-los em grupos que fossem coerentes e otimizassem a análise através da maior variação possível entre as categorias.

A estratégia do Programa de Redução de Danos facilitou trazer ao conhecimento das autoridades de saúde da cidade e do País essa população, até então invisível.

\section{Gráfico 3 - Distribuição de kits julho 2003 a agosto 2004}

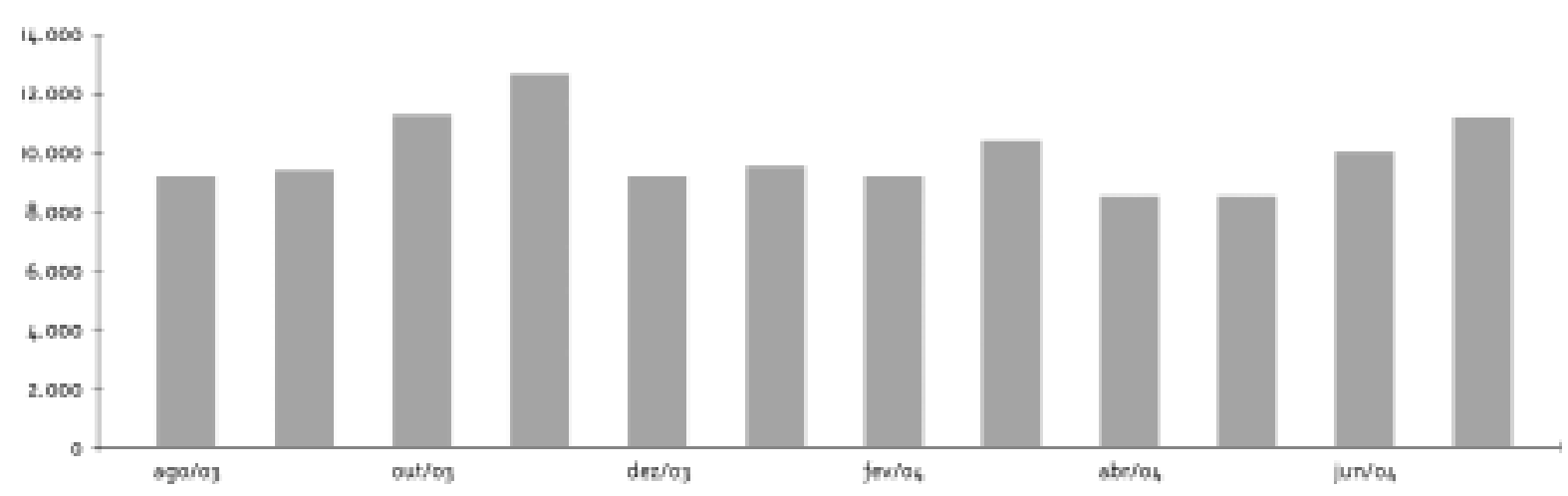

Fonte: PRD Sampa - Prefeitura Municipal de São Paulo

Portanto, a distribuição de seringas e kits é um facilitador para que o acesso e o vínculo com os UDIs possam ocorrer, configurando-se numa tecnologia a ser replicada cada vez mais nos serviços de saúde em geral, para que a Política Pública de redução de danos tenha o alcance necessário.

Mesmo serviços de saúde em Aids, que têm hábito de trabalhar com travestis, profissionais do sexo, HSH (homens que fazem sexo com homens) e outras populações específicas com HIV e Aids, não reconheciam a existência de UDIs entre seus clientes e nem sequer a existência deles na população. O que se configura em uma contradição: a notificação de Aids gerada por essas unidades aponta a importância de UDIs na transmissão do HIV na cidade de São Paulo.

$O$ indicador que aponta para a necessidade de ampliação das ações é o alto número de distribuição de seringas em todas as regiões de SP para um grande número de usuários. Há, portanto, muitos UDIs na ativa e estratégias de aproximação devem ser implantadas nos CAPS ad, por serem uma das vias de acesso aos serviços de saúde.

\section{Discussões - Análise das Categorias}

\section{Informação e formação}

O Treinamento Regionalizado mostrou-se a melhor resposta às necessidades de informação e de formação tanto dos trabalhadores da área, quanto da comunidade assistida. Com isso foi possível iniciar a disponibilização de insumos para os UDIs.

Nessa capacitação, há uma etapa fundamental que é a sensibilização dos próprios usuários de drogas não-injetáveis e das equipes de saúde, pois, há uma 
rejeição aos UDIs nos serviços. Portanto, é uma etapa de grande importância para as unidades que afirmam não ter UDIs que, só após treinamento, passam a reconhecer e acolher a demanda referenciada.

As estratégias de implantação baseadas nas experiências dos serviços de Aids passam a ser ampliadas e paulatinamente iniciadas nos CAPS ad. Algumas técnicas bem-sucedidas são utilizadas na implantação de RD em nossos serviços, por exemplo: disponibilização dos kits na recepção - o indicador de que população-alvo (UDI) existe é a retirada desses kits e, posteriormente, pode-se fazer o levantamento dessa população.

Outra estratégia utilizada é deixar os kits com filipetas para saber o que usuários, familiares e funcionários pensam sobre essa disponibilização. O levantamento dessas opiniões aponta para o nível de aceitação, ou não, do Programa de RD e o grau de facilidade ou dificuldade a ser enfrentado em sua implantação.

A formação dessas equipes e usuários dos serviços tem como objetivo desconstruir a resistência baseada no senso comum e associada a informações inadequadas e preconceitos contra usuários de drogas, tais como:

- Medo: de seu comportamento agressivo, de traficantes de drogas, de violência, de problemas com a polícia;

- Incompreensão sobre a distribuição de kits em um serviço de saúde pública;

- Suposição de que a intervenção possa estimular o uso de drogas ou mesmo criar a própria demanda.

\section{Parcerias}

Antes de se desenvolver um programa de RD, há necessidade de se implantar a Política Nacional de Drogas na qual RD é um dos componentes. Para que haja a inserção total desta Política está sendo necessário um processo que inclui a formação de uma sólida parceria com os serviços de Aids.

Essa parceria permite que à filosofia do CAPS ad, que é de tratamento da dependência química, sejam incorporadas estratégias sob a ótica da redução de danos. Isto, muitas vezes, cria um paradoxo para aqueles profissionais que tiveram formação ou experiência profissional dentro de um paradigma tradicional ou que não tenham a visão da Saúde Pública.

Isto porque o paradigma tradicional, através do qual o técnico recebeu sua formação, vê a cura como sinônimo de abstinência. Há, portanto, um tempo de latência para que esse choque de paradigmas seja absorvido. A abstinência não ser o foco do tratamento é um dos diferenciais dos serviços oferecidos pelos CAPS ad e premissa fundamental e norteadora dessas estratégias inovadoras.

As estratégias comunitárias de acesso ao UDI incluem parceria com outras instituições e ONGs facilitadoras do trabalho e da inserção da RD nas intervenções realizadas. O trabalho em conjunto com líderes comunitários favorece a identificação de locais que a comunidade refere como pontos de encontro de UDIs e que, sem a ajuda de pessoas que conhecem bem sua região, não seria possível atingi-los, por serem inacessíveis a quem não pertence à comunidade.

Uma parceria fundamental é a sensibilização da Polícia Militar, feita de forma regionalizada para ser mais eficiente. 0 procedimento ocorre através de visitas às Delegacias e às autoridades para apresentação da Lei da Redução de Danos do Estado de n $9.75^{8}$ de 17 de setembro de 1997, o que tem minimizado problemas e permitido a ampliação das ações.

\section{Sistemática de trabalho das equipes}

Nos serviços de Aids, o conceito de redução de danos discutido não deveria se limitar à distribuição de seringas. Ao contrário, a questão central era transformar os serviços de saúde pública em locais adequados para atender usuários de drogas. Esse olhar foi incorporado nos treinamentos para os CAPS ad, pois, facilitam o entendimento de como difundir estratégias de disponibilização de kits.

A pessoa do redutor de danos é de grande valia como articulador entre a comunidade, o PRD e os CAPS ad. Como são pessoas provindas de suas comunidades, quase sempre sem formação profissional especializada, apresentam problemas de adaptação às normas de trabalho. Essas dificuldades exigem das equipes programar supervisões e grupos de apoio psicológico e social, facilitando assim a inclusão desses novos integrantes.

A sistemática de trabalho das equipes de tratamento da dependência química (DQ) difere dos serviços de Aids, havendo necessidade de tecnologia específica para CAPS ad. Implantou-se um projeto piloto na Unidade do Jabaquara na busca de uma metodologia que facilitasse o desenvolvimento de estratégias de aproximação e acolhimento dos UDIs, mesmo que não 
inseridos no tratamento. Ou seja, distribuição de kits em um centro de tratamento torna-se um desafio se configurando num paradoxo.

Conceituar e propiciar a incorporação de estratégias inovadoras remetem ao cuidar, que inclui, ou não, o tratamento específico para a dependência química. O caminho para a implantação desse processo é longo e as unidades e suas equipes têm ritmos diferentes de assimilação do conceito. Alguns profissionais e também aqueles com formação em saúde pública podem ser os alavancadores das equipes para a aceitação do novo conceito de saúde .

Algumas unidades de saúde dizem que não há UDIs em seus serviços ou em seu território. Ensinar e sensibilizar a todos é importante - o acolhimento é a ferramenta fundamental ao cuidar e aproximar o UDI dos serviços de saúde. A distribuição de kits funciona como um intermediário dessa relação e, também, como via de acolhimento que vai além do insumo distribuído: é o cuidar que está em jogo nesse processo.

O envolvimento de todos da unidade de serviço, ao realizar um trabalho mais integrado, possibilita a humanização do atendimento e é o indicador de que o conceito de RD está sendo internalizado. Portanto, ao planejar a implantação de intervenções em RD, deve-se ter em mente que é um processo gradativo, longo e deve respeitar as diferenças das equipes e dos serviços.

\section{Inserção e pertencimento}

Essas equipes como um todo e, principalmente, os redutores de danos envolvem-se com os problemas de sua comunidade, sendo necessário um trabalho de fortalecimento desses profissionais para que a frustração - por não poder resolver tudo e atingir a todos, seja diluída e não impeça a continuidade da tarefa.

O projeto piloto foi desenhado para o CAPS ad Jabaquara, pela facilidade que seu grupo de técnicos apresenta em assimilar os novos conceitos e acolher os UDIs de forma mais isenta de preconceitos. Essa equipe preencheu os critérios de coesão e unidade que facilitam o acesso e a adesão dos UDIs, assim como consolidam a parceria com a comunidade e com os serviços existentes no bairro.

Portanto, também é fundamental ao acolher o UDI, a parceria com os serviços de DST/AIDS. Isto porque os redutores de danos integram essas equipes e são interlocutores que podem conduzí-los aos CAPS ad e tornam-se referência e são fundamentais.

\section{Mudanças e benefícios}

Por muito tempo, acreditou-se que os usuários de drogas - especialmente os usuários de drogas injetáveis fossem incapazes de modificar seu comportamento. Os programas de redução de danos têm mostrado que era uma falsa premissa, pois estes são, não apenas a forma mais eficaz de gerar saúde entre usuários de drogas, mas, principalmente, de dar à sociedade e aos governos uma metodologia eficiente para atuarem juntos, no resgate da cidadania de pessoas até então marginalizadas e discriminadas.

A redução de danos contribui significativamente para a construção de um modelo exitoso de prevenção e atuação mais democrático na área da saúde, no qual o saber circula entre os trabalhadores de saúde, os usuários dos serviços e a comunidade como um todo.

Propicia, também, o engajamento da comunidade, por meio de suas associações, organizações, redes de interação social, grupos e organizações religiosas, na discussão da problemática da Aids e do uso de drogas, garantindo a sustentabilidade das ações.

Observa-se que, quanto maior o envolvimento da comunidade, maiores são os benefícios primários efetividade e resultados das ações, e secundários para a população - melhoria de condições de vida, diminuição dos índices de violência, etc.

O protagonismo, ou seja, a participação de grupos de usuários e ex-usuários de drogas nas instâncias legítimas de discussão das políticas públicas de saúde tem possibilitado que as autoridades responsáveis pelo setor passem a delinear estratégias que efetivamente ampliem o acesso dessa população aos serviços de saúde. Tais serviços incluem o tratamento da dependência química, tratamento dos problemas decorrentes do uso da droga, como a Aids e outras doenças de transmissão sangüínea, além de permitir que os insumos necessários à prevenção - preservativos, material informativo e seringas descartáveis, sejam disponibilizados para aqueles que deles necessitam prioritariamente.

\section{Acolhimento e confiança}

Em um serviço médico ou em um programa em atenção a usuários de drogas, baseados nos princípios de $\mathrm{RD}$, os usuários de drogas não se sentem incapazes de se ajudar, mas são estimulados a uma participação ativa. 
Tal abordagem para cuidados com a saúde e o suporte social para os usuários de drogas inclui a percepção de seus direitos como cidadão, juntamente com a minimização da exclusão, mesmo aquela que resulta dos aspectos negativos de sua aparência e de sua conduta. A implantação do conceito de RD em um serviço de saúde pode encontrar resistência dos funcionários. Com um trabalho integrado da equipe e das coordenações é possível sensibilizá-los para posterior internalização desses conceitos.

Os profissionais já sensibilizados podem incorporar um conceito mais abrangente e isto permite aos mesmos incluir outras populações também vulneráveis em suas intervenções para minimizar os riscos sociais e à saúde.

Um fator de extrema importância é a garantia de que a distribuição dos kits nos centros de tratamento da DQ seja anônima e que não esteja vinculada como princípio à obrigatoriedade ao tratamento.

\section{Conclusões}

Implantar um Programa de Redução de Danos, mesmo que apoiado por Leis e assumido como Política Pública em nível Federal, Estadual e local é uma tarefa difícil e que exige criatividade, esforço conjunto e flexibilidade nos prazos de implementação. A Portaria no. 2.197, de 14 de outubro de 2004, do Ministro da Saúde que redefine e amplia a atenção integral para usuários de álcool e outras drogas, no âmbito do Sistema Único de Saúde - SUS, embasa as ações dos serviços de Saúde Mental, preconizando a lógica de redução de danos, por ser uma estratégia bem sucedida para as ações desenvolvidas no sistema de saúde público.

$\mathrm{Na}$ apresentação dos antecedentes deste estudo percebe-se que ao se focar um trabalho em UDIs, estamos trabalhando com uma população de acesso complicado e difícil de ser acolhida, mesmo em serviços de Aids.

Para que a implantação ocorra em outros serviços de saúde, além da Aids, como a rede básica de saúde e serviços de tratamento da dependência química, toda a tecnologia adquirida nos PRDs precisa ser adaptada e monitorada para avaliação de resultados.

A partir da parceria com o PRD ter sido consolidada e treinamentos realizados foi possível conhecer a dimensão da tarefa a ser cumprida. Foi também um marco para que se delineasse um plano de trabalho flexível e completo suficiente para que abarcasse dificuldades e demandas que surgissem no decorrer da realização das tarefas.

Após treinamento dos CAPS ad, grupos de fortalecimento das ações começaram a ser realizados e um Projeto Piloto tornou-se uma necessidade, pois, permitia a observação direta dos resultados das intervenções realizadas.

Portanto, ação de distribuição de kits não é tão simples como parecia inicialmente, pois envolve inúmeros procedimentos que se relacionam a questões anteriores à implantação da disponibilização de seringas. E é um tipo de procedimento que precisa ser cuidadoso - o que pode levar tempo - pois, conforme o local, desperta polêmica na comunidade, principalmente nas regiões onde se costuma afirmar aqui não tem UDI ou, se aparecem UDIs, afirmam que estão migrando para a região porque há oferta de kits e que caso não houvesse essa intervenção os UDIs não estariam naquela região.

É um processo muito complexo porque envolve discriminação, direitos humanos e cidadania numa temática que desperta nos profissionais medo e preconceito. Isto, provavelmente por lidar com drogas ilícitas algo proibido - e os profissionais e o pessoal de suporte temem ser cúmplices, ao invés de curar o dependente.

Para que isso não ocorra há necessidade da ampliação do conceito de tratamento para cuidar do UDI. E uma das ações possíveis é a disponibilização dos kits e seringas para que sua saúde e da comunidade seja preservada.

Outro fator de grande relevância é que redução de danos não é só para o UDI, nem só para os usuários de drogas ilícitas, pois, as ações abrangem o uso de álcool, fazendo com que a complexidade do trabalho seja maior.

Para se iniciar um trabalho sob a ótica da redução de danos num serviço público, principalmente de tratamento da dependência química, é essencial que a equipe primeiro internalize a premissa de que abstinência não é o foco primordial de seu trabalho.

A partir da apropriação desse conceito mais abrangente e da mudança de foco do tratamento, as equipes de Saúde Mental do CAPS ad foram treinadas e fortalecidas em novas tecnologias para subsidiar suas intervenções. O serviço de Saúde Mental - Álcool 
e Drogas do município de São Paulo, em parceria com a DST/AIDS vem seguindo o seguinte modelo de intervenção:

- Redução de danos é um instrumental abrangente embasado em conceitos da Saúde Pública e , portanto, replicável para todos os usuários dos serviços e não só para o UDI. Implica em considerar que a abstinência não é o foco do cuidado ao usuário que é acessado ou chega ao serviço, seja ele UDI ou não.

- A mudança de padrão de consumo de droga é uma meta, pois, passar de um padrão ou forma de uso de drogas de maior risco, para um de menor risco, pode ser uma meta demandada pelo próprio usuário ou estabelecida em conjunto com os profissionais da equipe vinculados a esse usuário.

- Outro passo a ser internalizado pelas equipes é o das terapias de substituição, pois, como a droga ilegal de uso mais difundido no Brasil é a cocaína, não há droga de substituição reconhecida. Portanto, substituir um padrão de consumo de injetável por aspirado, por exemplo, ou a cocaína/crack pela maconha, constituise em um recurso possível para a minimização dos riscos a esse usuário e à sua rede social.

- A prevenção de overdose ou de intoxicação aguda quando assimilada pelas equipes e, assim passível de ser transmitida aos usuários, pode ser uma forma de redução de graves danos à saúde ou mesmo morte.

- Oferecimento de insumos de prevenção, ou seja, dos kits para os UDIs, assim como kits específicos para usuários de outras drogas, como a oferta de cachimbos aos usuários de crack, ou canudos descartáveis para os que utilizam cocaína aspirada.

Em toda a rede de CAPS ad de São Paulo o primeiro item está consolidado. Os outros quatro itens estão paulatinamente sendo atingidos, respeitando o momento da equipe e sua maior ou menor facilidade para incorporar os conceitos.

Na Saúde Mental, a Redução de Danos pode ter a função de auxiliar a revisão do modelo de tratamento do dependente químico por exigir: um tipo de formação dos profissionais, a reorganização do serviço para receber o usuário de drogas (acolhimento) e locais para passagens rápidas (para adesão ao tratamento, por exemplo); além de ser referência para os redutores de danos ao garantir encaminhamento a serviços.
Outra função importante dessa parceria e cooperação é a supervisão da equipe do $\mathrm{PRD}$, uma vez que a Saúde Mental, historicamente, trabalha com supervisão de profissionais e esta prática foi incorporada aos programas de redução de danos.

\section{Lições aprendidas}

1. Não há um modelo fechado de implantação de RD em CAPS, nem um modelo totalmente replicável: há diretrizes básicas, mas os procedimentos têm de ser apropriados e levam-se meses, muitas vezes, para que o tema comece a ser incorporado na rotina do serviço.

2. Equipe é fundamental. E as características fundamentais dessa equipe são: coesão, aceitação, conhecimento, compreensão abrangente da intervenção. Quando se fala em equipe se fala desde o porteiro ou segurança, daqueles que estão na recepção, na porta de entrada, ou seja, o pessoal de suporte, além da equipe técnica.

3. Há necessidade de grande integração com os líderes comunitários para que intercedam quando necessário for.

4. 0 treinamento regionalizado e supervisão institucional mostraram-se fundamentais no intuito de superar preconceitos e, realmente, inserir os UDIs. Um benefício dessas ações é o fato de que os profissionais começam a perceber que havia UDIs nos serviços, mas que não apareciam, eram invisíveis, pois, sentiam-se não-acolhidos. Esta mudança na postura dos profissionais permitiu e continua facilitando a visibilidade dos mesmos.

5. Um fator que muito vem auxiliando na implantação das intervenções é a existência da Lei que assegura a integridade do funcionário e do redutor de danos, garantindo a continuidade das ações.

6. CAPS tem um território definido e pode ser a referência para o PRD encaminhar além da contribuição que pode oferecer, pois, tem equipe multidisciplinar para dar suporte ao PRD e ser a referência de acompanhamento das difíceis questões emocionais e técnicas que os redutores enfrentam em seu dia a dia no campo. Pois, parceria sempre é uma via de mão dupla.

7. Acessar o UDI no campo é uma forma de aproximálo dos serviços de saúde. Há necessidade de que o UDI desconstrua seu preconceito e aceite que buscar o ser- 
viço de tratamento, não implica na necessidade de ficar e estar abstinente, há necessidade de se consolidar um caminho que se inicie pelo acesso, conduza a sensibilização para um novo conceito e, posteriormente, vincule esse cidadão ao serviço de saúde.

\section{Referências}

BRASIL. Ministério da Saúde. Coordenação Nacional de DST e Aids. Manual de redução de danos. Brasília, DF, 2001a. 120 p.

BRASIL. Ministério da Saúde. A política do Ministério da Saúde para a atenção integral a usuários de álcool e outras drogas. 2. ed. revisada e ampliada. Brasília, DF, 2004a. 60 p.

BRASIL. Ministério da Saúde. Legislação em saúde mental: 1990-2004. 5. ed. ampliada. Brasília, DF, 2004b. 340 p. (Legislação de Saúde).

Saúde mental - Álcool e drogas. Site desenvolvido pelo Ministério da Saúde. Brasília, DF. Disponível em: <http://portal.saude.gov.br/portal/sas/mental/ visualizar_texto.cfm?idtxt $=22919 \&$ janela $=1>$. Acesso em: o6 jun 2004.
BRASIL. Sistema Único de Saúde. Conselho Nacional de Saúde. Comissão Organizadora da III CNSM. Relatório Final da III Conferência Nacional de Saúde Mental: Brasília, 11 a 15 de dezembro de 2001b. Brasília, DF: Conselho Nacional de Saúde/Ministério da Saúde, 2002. 213p.

CAIAFFA, W. T.et al. Projeto AJUDE-Brasil: avaliação epidemiológica dos usuários de drogas injetáveis dos Projetos de Redução de Danos (PRD) apoiados pelo CNDST/Aids. Ministério da Saúde, 2001. Disponível em: <http://www.aids.gov.br/final/biblioteca/avaliacao6/ ajud.htm>. Acesso em: 15 fev 2004.

ONU - Organização das Nações Unidas. Declaração Universal dos Direitos Humanos. Disponível em: <http:// www.mj.gov.br/sedh/ct/legis_intern/ddh_bib_inter universal.htm>. Acesso em: 15 fev 2004.

ONU - Organização das Nações Unidas. Posição oficial do Sistema das Nações Unidas: prevenção da transmissão do HIV entre usuários de drogas. Anexo do Relatório da $8^{a}$ Sessão do Sub-comitê sobre Controle de Drogas da ACC. 200o. Disponível em: <http://www.aids. gov.br/final/prevencao/udi_nacoes_unidas.doc $>$. Acesso em: o6 jun 2004 . 